\title{
PROJECTIVITY AND FLATNESS OVER THE ENDOMORPHISM RING OF A FINITELY GENERATED MODULE
}

\author{
S. CAENEPEEL and T. GUÉDÉNON
}

Received 7 October 2003

Let $A$ be a ring and $\Lambda$ a finitely generated $A$-module. We give necessary and sufficient conditions for projectivity and flatness of a module over the endomorphism ring of $\Lambda$.

2000 Mathematics Subject Classification: 16D40, 16W30.

1. Introduction. Suppose that a group $G$ acts as a group of automorphisms on a ring $R$. Jøndrup [11] has studied when $R$ is projective as an $R^{G}$-module. García and Del Río [8] have continued Jøndrup's work, and gave necessary and sufficient conditions for the flatness and projectivity of $R$ as an $R^{G}$-module. In [10], the second author generalized the results from [8] in the following way. Let $k$ be a commutative ring, $H$ a Hopf algebra over $k$, and $\Lambda$ a left $H$-module algebra. Then we can consider the smash product $\Lambda \# H$ and the subring of invariants $\Lambda^{H}$. Then we can give necessary and sufficient conditions for the projectivity and flatness over $\Lambda^{H}$ of a left $\Lambda \# H$-module $P$. The results from [8] are recovered if we take $k=\mathbb{Z}, H=k G$, and $P=R$.

The authors discovered recently that the same methods can be applied to the following situation. Let $j: S \rightarrow \Lambda$ be a ring morphism, and consider the centralizer $\Lambda^{S}=\{b \in$ $\Lambda \mid b j(s)=j(s) b$, for all $s \in S\}$. Then we can give necessary and sufficient conditions for projectivity and flatness over $\Lambda^{S}$ of an $(S, \Lambda)$-bimodule.

In fact, both results are special cases of a more general result which happens to be very elementary. Let $\Lambda$ be a finitely generated module over a ring $A$, and $B$ the endomorphism ring of $\Lambda$. Adapting the techniques developed in [8, 10], we give, in Section 2, some necessary and sufficient conditions for projectivity and flatness of a $B$-module.

It turns out that in our two motivating examples, $\Lambda$ is a cyclic module. Such modules appeared recently in [6] and are obtained by considering a ring morphism with a grouplike functional. This will be explained in Section 3.

2. Main result. Let $A$ be an associative ring with unit, and $\Lambda \in \mu_{A}$, where $\mu_{A}$ is the category of right $A$-modules and right $A$-linear maps. All our results hold for left modules as well, but the formulas are slightly more elegant in the right-handed case, a consequence of the fact that we write from left to right. Let $B=\operatorname{End}_{A}(\Lambda)$. Then $\Lambda$ is a $(B, A)$-bimodule; the left action is just evaluation: $b \cdot \lambda=b(\lambda)$, for all $b \in B$ and $\lambda \in \Lambda$. Then we have an adjoint pair of functors $(F, G)$ connecting the categories of 
right $B$-modules and right $A$-modules:

$$
F=\bullet \otimes_{B} \Lambda: M_{B} \longrightarrow M_{A}, \quad G=\operatorname{Hom}_{A}(\Lambda, \bullet): \mu_{A} \longrightarrow M_{B}
$$

For $M \in M_{A}, G(M)=\operatorname{Hom}_{A}(\Lambda, M)$ is a right $B$-module, by $(f \cdot b)(\lambda)=f(b \lambda)$. The unit and counit of the adjunction are defined as follows, for $M \in \mathcal{M}_{A}, N \in \mathcal{M}_{B}$ :

$$
\begin{array}{ll}
\eta_{N}: N \longrightarrow \operatorname{Hom}_{A}\left(\Lambda, N \otimes_{B} \Lambda\right), & \eta_{N}(n)(\lambda)=n \otimes \lambda, \\
\varepsilon_{M}: \operatorname{Hom}_{A}(\Lambda, M) \otimes_{B} \Lambda \longrightarrow M, & \varepsilon_{M}(f \otimes \lambda)=f(\lambda) .
\end{array}
$$

The adjointness property then means that we have

$$
G\left(\varepsilon_{M}\right) \circ \eta_{G(M)}=I_{G(M)}, \quad \varepsilon_{F(N)} \circ F\left(\eta_{N}\right)=I_{F(N)} .
$$

In particular, $\eta_{G(M)}$ is monic for every $M \in \mathcal{M}_{A}$.

Recall from [13] that $\Lambda \in M_{A}$ is called semi- $\Sigma$-projective if the functor $\operatorname{Hom}_{A}(\Lambda, \bullet)$ : $\mu_{A} \rightarrow \underline{\underline{\mathrm{Ab}}}$ sends an exact sequence in $\mu_{A}$ of the form

$$
\Lambda^{(J)} \longrightarrow \Lambda^{(I)} \longrightarrow M \longrightarrow 0
$$

to an exact sequence in $\underline{\underline{\mathrm{Ab}}}$. Obviously, a projective module is semi- $\Sigma$-projective.

LEMMA 2.1. Let $\Lambda$ be a finitely generated right $A$-module, and $B=\operatorname{End}_{A}(\Lambda)$. For every set I,

(1) the natural map $\kappa: B^{(I)}=\operatorname{Hom}_{A}(\Lambda, \Lambda)^{(I)} \rightarrow \operatorname{Hom}_{A}\left(\Lambda, \Lambda^{(I)}\right)$ is an isomorphism;

(2) $\varepsilon_{\Lambda^{(I)}}$ is an isomorphism;

(3) $\eta_{B^{(I)}}$ is an isomorphism;

(4) if $\Lambda$ is semi- $\Sigma$-projective as a right A-module, then $\eta$ is a natural isomorphism; in other words, the induction functor $F=\bullet \otimes_{A} \Lambda$ is fully faithful.

Proof. (1) is an easy exercise.

(2) It is straightforward to check that the canonical isomorphism $B^{(I)} \otimes_{B} \Lambda \cong \Lambda^{(I)}$ is the composition of the maps

$$
B^{(I)} \otimes_{B} \Lambda \stackrel{\kappa \otimes I_{\Lambda}}{\longrightarrow} \operatorname{Hom}_{A}\left(\Lambda, \Lambda^{(I)}\right) \otimes_{B} \Lambda \stackrel{\varepsilon_{\Lambda^{(I)}}}{\longrightarrow} \Lambda^{(I)}
$$

and it follows from (1) that $\varepsilon_{\Lambda^{(I)}}$ is an isomorphism.

(3) Putting $M=\Lambda^{(I)}$ in (2.3), we find

$$
\operatorname{Hom}_{A}\left(\Lambda, \varepsilon_{\Lambda^{(I)}}\right) \circ \eta_{\operatorname{Hom}_{A}\left(\Lambda, \Lambda^{(I)}\right)}=I_{\operatorname{Hom}_{A}\left(\Lambda, \Lambda^{(I)}\right)} .
$$

From (2), it follows that $\operatorname{Hom}_{A}\left(\Lambda, \varepsilon_{\Lambda^{(I)}}\right)$ is an isomorphism and from (1), it follows that $\operatorname{Hom}_{A}\left(\Lambda, \Lambda^{(I)}\right) \cong B^{(I)}$, hence $\eta_{B^{(I)}}$ is an isomorphism.

(4) follows from [13, Theorem 2.1]. We include a short proof for the sake of completeness. Take a free resolution $B^{(J)} \rightarrow B^{(I)} \rightarrow N \rightarrow 0$ of a right $B$-module $N$. $\eta$ is natural, so 
we have a commutative diagram

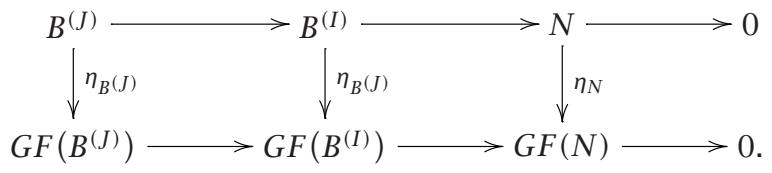

The top row is exact, the bottom row is exact, since $G F\left(B^{(I)}\right)=\operatorname{Hom}_{A}\left(\Lambda, \Lambda^{(I)}\right)$ and $\Lambda$ is semi- $\Sigma$-projective. By (3), $\eta_{B^{(I)}}$ and $\eta_{B^{(J)}}$ are isomorphisms, and it follows from the five lemma that $\eta_{N}$ is an isomorphism.

We can now give equivalent conditions for the projectivity and flatness of $P \in M_{B}$.

THEOREM 2.2. Assume that $\Lambda \in \mu_{A}$ is finitely generated and let $B=\operatorname{End}_{A}(\Lambda)$. For $P \in \mathcal{M}_{B}$, the following statements are considered:

(1) $P \otimes_{B} \Lambda$ is projective in $\mu_{A}$ and $\eta_{P}$ is injective;

(2) $P$ is projective as a right $B$-module;

(3) $P \otimes_{B} \Lambda$ is a direct summand in $\mu_{A}$ of some $\Lambda^{(I)}$, and $\eta_{P}$ is bijective;

(4) there exists $Q \in M_{A}$ such that $Q$ is a direct summand of some $\Lambda^{(I)}$, and $P \cong$ $\operatorname{Hom}_{A}(\Lambda, Q)$ in $\mu_{B}$;

(5) $P \otimes_{B} \Lambda$ is a direct summand in $\mu_{A}$ of some $\Lambda^{(I)}$.

Then $(1) \Rightarrow(2) \Leftrightarrow(3) \Leftrightarrow(4) \Rightarrow(5)$. If $\Lambda$ is semi- $\sum$-projective (in particular, $\Lambda$ is quasi-projective, see [1, Proposition 1.8]) as a right $A$-module, then (5) $\Rightarrow(3)$; if $\Lambda$ is projective as a right A-module, then (2) $\Rightarrow(1)$.

Proof. (2) $\Rightarrow(3)$. If $P$ is projective as a right $B$-module, then we can find a set $I$ and $P^{\prime} \in M_{B}$ such that $B^{(I)} \cong P \oplus P^{\prime}$. Then, obviously, $\Lambda^{(I)} \cong B^{(I)} \otimes_{B} \Lambda \cong\left(P \otimes_{B} \Lambda\right) \oplus\left(P^{\prime} \otimes_{B} \Lambda\right)$. $\eta$ is a natural transformation, so we have a commutative diagram

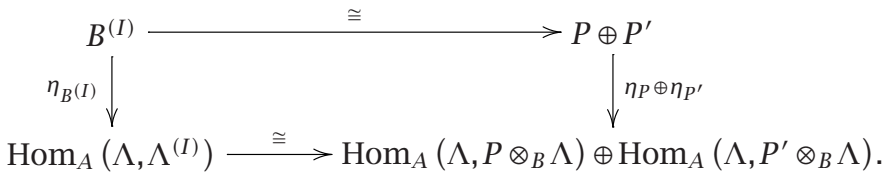

From the fact that $\eta_{B^{(I)}}$ is an isomorphism, it follows that $\eta_{P}$ (and $\eta_{P^{\prime}}$ ) are isomorphisms.

(3) $\Rightarrow$ (5). Take $Q=P \otimes_{B} \Lambda$.

(4) $\Rightarrow(2)$. If $f: \Lambda^{(I)} \rightarrow Q$ is a split epimorphism in $\mu_{A}$, then

$$
\operatorname{Hom}_{A}(\Lambda, f): \operatorname{Hom}_{A}\left(\Lambda, \Lambda^{(I)}\right) \cong B^{(I)} \longrightarrow \operatorname{Hom}_{A}(\Lambda, Q) \cong P
$$

is also split surjective, hence $P$ is projective as a right $B$-module.

$(4) \Rightarrow(5)$ is trivial.

(5) $\Rightarrow$ (3) under the assumption that $\Lambda$ is semi- $\sum$-projective; this follows immediately from Lemma 2.1(4).

$(1) \Rightarrow(2)$. Take an epimorphism $f: B^{(I)} \rightarrow P$ in $\mu_{B}$. Then $f \otimes_{B} I_{\Lambda}: \Lambda^{(I)} \rightarrow P \otimes_{B} \Lambda$ is also surjective, and split in $\mathcal{M}_{A}$, since $P \otimes_{B} \Lambda$ is projective. Consider the commutative 
diagram

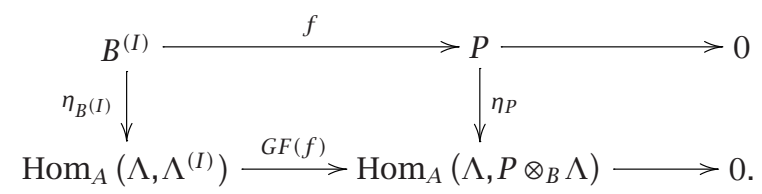

The bottom row is split exact since any functor, in particular, $\operatorname{Hom}_{A}(\Lambda, \bullet)$, preserves split exact sequences. $\eta_{B^{(I)}}$ is an isomorphism, by Lemma 2.1, and diagram chasing tells us that $\eta_{P}$ is surjective. By assumption, $\eta_{P}$ is injective, so $\eta_{P}$ is bijective, and the top row is isomorphic to the bottom row and therefore split. Thus $P \in M_{B}$ is projective.

$(2) \Rightarrow(1)$. If $\Lambda$ is projective in $\mu_{A}$, then $\Lambda$ is a direct summand of $A^{(J)}$ in $\mu_{A}$ for some set $J$. Now $P \otimes_{B} \Lambda$ is a direct summand of $\Lambda^{(I)}$ and also of $A^{(J \times I)}$, so $P \otimes_{B} \Lambda \in M_{A}$ is projective. Since (2) implies (3), it follows that $\eta_{P}$ is injective.

The Govorov-Lazard theorem $[9,12]$ states that $M \in \mu_{A}$ is flat if and only if it is the inductive limit of a directed system of free modules of finite type. Theorem 2.3 can be viewed as a generalization of this classical result.

THEOREM 2.3. Assume that $\Lambda \in M_{A}$ is finitely presented and let $B=\operatorname{End}_{A}(\Lambda)$. For $P \in \mathcal{M}_{B}$, the following assertions are equivalent:

(1) $P$ is flat as a right $B$-module;

(2) $P \otimes_{B} \Lambda=\underline{\lim } Q_{i}$, where $Q_{i} \cong \Lambda^{n_{i}}$ in $\mu_{A}$ for some positive integer $n_{i}$, and $\eta_{P}$ is bijective;

(3) $P \otimes_{B} \Lambda=\underline{\lim } Q_{i}$, where $Q_{i} \in M_{A}$ is a direct summand of some $\Lambda^{(I)}$, and $\eta_{P}$ is bijective;

(4) there exists $Q=\underline{\lim _{\longrightarrow}} Q_{i} \in M_{A}$, such that $Q_{i} \cong \Lambda^{n_{i}}$ for some positive integer $n_{i}$ and $\operatorname{Hom}_{A}(\Lambda, Q) \cong P$ in $M_{B}$

(5) there exists $Q=\lim _{\longrightarrow} Q_{i} \in \mathcal{M}_{A}$, such that $Q_{i}$ is a direct summand of $\Lambda^{(I)}$ as a right $A$-module and $\operatorname{Hom}_{A}(\Lambda, Q) \cong P$ in $M_{B}$.

If $\Lambda$ is semi- $\Sigma$-projective as a right $A$-module, then these conditions are also equivalent to conditions (2) and (3) without the assumption that $\eta_{P}$ is bijective.

Proof. (1) $\Rightarrow(2) . P=\underline{\lim } N_{i}$, with $N_{i}=B^{n_{i}}$. Take $Q_{i}=\Lambda^{n_{i}}$, then

$$
\stackrel{\lim }{\longrightarrow} Q_{i} \cong \underline{\lim }\left(N_{i} \otimes_{B} \Lambda\right) \cong\left(\lim _{\longrightarrow} N_{i}\right) \otimes_{B} \Lambda \cong P \otimes_{B} \Lambda .
$$

Consider the following commutative diagram:

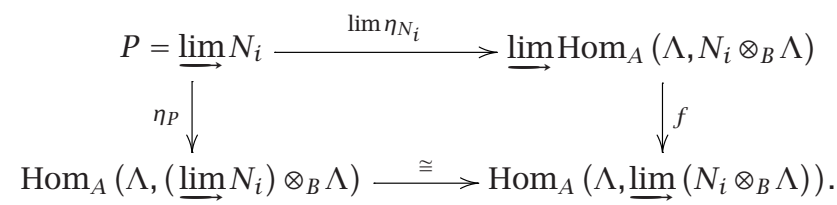

By Lemma 2.1, the $\eta_{N_{i}}$ are isomorphisms; the natural homomorphism $f$ is an isomorphism because $\Lambda$ is finitely presented. Hence $\eta_{P}$ is an isomorphism. 
(2) $\Rightarrow(3)$ and $(4) \Rightarrow(5)$ are obvious.

(2) $\Rightarrow(4)$ and (3) $\Rightarrow(5)$. Put $Q=P \otimes_{B} \Lambda$. Then $\eta_{P}: P \rightarrow \operatorname{Hom}_{A}\left(\Lambda, P \otimes_{B} \Lambda\right)$ is the required isomorphism.

$(5) \Rightarrow(1)$. We have a split exact sequence

$$
0 \longrightarrow N_{i} \longrightarrow P_{i}=\Lambda^{(I)} \longrightarrow Q_{i} \longrightarrow 0 .
$$

Consider the following commutative diagram

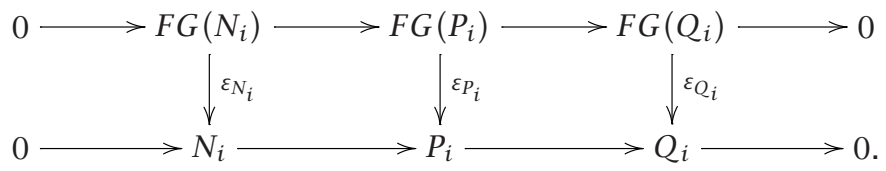

We know, from Lemma 2.1, that $\varepsilon_{P_{i}}$ is an isomorphism. Both rows in the sequence are split exact, so it follows that $\varepsilon_{N_{i}}$ and $\varepsilon_{Q_{i}}$ are also isomorphisms. Next, consider the commutative diagram

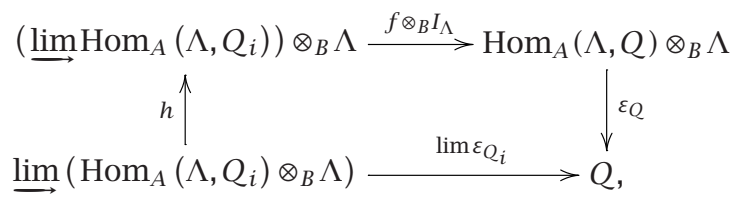

where $h$ and $f$ are the natural homomorphisms. $h$ is an isomorphism because $\bullet \otimes_{B} \Lambda$ preserves inductive limits, and $f$ is an isomorphism because $\Lambda \in M_{A}$ is finitely presented. $\lim \varepsilon_{Q_{i}}$ is an isomorphism because every $\varepsilon_{Q_{i}}$ is an isomorphism, so it follows that $\varepsilon_{Q}$ is an isomorphism. From (2.3), we deduce

$$
\operatorname{Hom}_{A}\left(\Lambda, \varepsilon_{Q}\right) \circ \eta_{\operatorname{Hom}_{A}(\Lambda, Q)}=I_{\operatorname{Hom}_{A}(\Lambda, Q)} .
$$

$\operatorname{Hom}_{A}\left(\Lambda, \varepsilon_{Q}\right)$ is an isomorphism, so $\eta_{\operatorname{Hom}_{A}(\Lambda, Q)}$ is also an isomorphism, and, since $\operatorname{Hom}_{A}(\Lambda, Q) \cong P, \eta_{P}$ is an isomorphism. Consider the isomorphisms

$$
\begin{gathered}
P \stackrel{\eta_{P}}{\longrightarrow} \operatorname{Hom}_{A}\left(\Lambda, P \otimes_{B} \Lambda\right) \stackrel{\cong}{\longrightarrow} \operatorname{Hom}_{A}\left(\Lambda, \operatorname{Hom}_{A}(\Lambda, Q) \otimes_{B} \Lambda\right) \\
\stackrel{\operatorname{Hom}\left(\Lambda, \varepsilon_{Q}\right)}{\longrightarrow} \operatorname{Hom}_{A}(\Lambda, Q) \stackrel{f}{\longrightarrow} \underset{\lim }{\longrightarrow} \operatorname{Hom}_{A}\left(\Lambda, Q_{i}\right) .
\end{gathered}
$$

It follows from Lemma 2.1 that $\operatorname{Hom}_{A}\left(\Lambda, P_{i}\right) \cong B^{\left(I_{i}\right)}$ is projective as a right $B$-module, hence $\operatorname{Hom}_{A}\left(\Lambda, Q_{i}\right)$ is also projective as a right $B$-module, and we conclude that $P \in M_{B}$ is flat.

The final statement is an immediate consequence of Lemma 2.1(4).

REMARK 2.4. To $\Lambda \in M_{A}$, we can associate a Morita context (cf. [2]), namely, ( $A, B, \Lambda$, $\left.\Lambda^{*}=\operatorname{Hom}_{A}(\Lambda, A), \tau, \mu\right)$ with connecting maps

$$
\begin{gathered}
\tau: \Lambda \otimes_{A} \Lambda^{*} \longrightarrow B, \quad \tau\left(\lambda \otimes \lambda^{*}\right)(\mu)=\lambda \lambda^{*}(\mu), \\
\mu: \Lambda^{*} \otimes_{B} \Lambda \longrightarrow A, \quad \mu\left(\lambda^{*} \otimes \lambda\right)=\lambda^{*}(\lambda) .
\end{gathered}
$$


$\tau$ is surjective (and a fortiori bijective) if and only if $\Lambda$ is finitely generated and projective as a right $A$-module. In this situation, the natural transformation

$$
\gamma: G_{1}=\bullet \otimes_{A} \operatorname{Hom}_{A}(\Lambda, A) \longrightarrow G=\operatorname{Hom}_{A}(\Lambda, \bullet)
$$

with $\gamma_{M}\left(m \otimes_{A} \lambda^{*}\right)(\lambda)=m \lambda^{*}(\lambda)$ is a natural isomorphism.

For any $N \in \mathcal{M}_{B}$, we easily compute that

$$
\gamma_{N \otimes_{B} \Lambda}=\eta_{N} \circ\left(I_{N} \otimes T\right) .
$$

We can conclude that $\eta$ is a natural isomorphism if $\Lambda$ is finitely generated and projective as a right $A$-module. This is an alternative proof of a special case of Lemma 2.1.

3. Main example. Let $\Lambda$ and $A$ be rings, $i: \Lambda \rightarrow A$ a ring morphism, and $\chi: A \rightarrow \Lambda$ a right grouplike functional. This means that $\chi$ satisfies the following properties, for all $a, b \in A$ (see [6]):

(1) $\chi$ is right $\Lambda$-linear;

(2) $\chi(\chi(a) b)=\chi(a b)$;

(3) $x\left(1_{A}\right)=1_{\Lambda}$.

Then $\Lambda \in \mathcal{M}_{A}$, with action

$$
\lambda-a=\chi(\lambda a)
$$

$\Lambda$ is finitely generated, in fact it is a cyclic module, since

$$
\lambda=\chi\left(1_{A}\right) \lambda=\chi\left(1_{A} \lambda\right)=\chi\left(1_{A} \lambda 1_{\Lambda}\right)=1_{\Lambda}-\left(1_{A} \lambda\right)
$$

for all $\lambda \in \Lambda$. It is easy to verify that

$$
\operatorname{Hom}_{A}(\Lambda, M) \cong M^{A}=\{m \in M \mid m a=m \chi(a), \forall a \in A\} .
$$

In particular,

$$
B=\operatorname{Hom}_{A}(\Lambda, \Lambda) \cong \Lambda^{A}=\{b \in \Lambda \mid b \chi(a)=\chi(b a), \forall a \in A\} .
$$

We can apply Theorems 2.2 and 2.3, and we find necessary and sufficient conditions for $P \in \mathcal{M}_{B}$ to be projective or flat. Observe that $\Lambda$ is semi- $\Sigma$-projective if the functor $(\bullet)^{A}$ sends an exact sequence $\Lambda^{(J)} \rightarrow \Lambda^{(I)} \rightarrow M \rightarrow 0$ in $\mathcal{M}_{A}$ to a free resolution $B^{(J)} \rightarrow B^{(I)} \rightarrow$ $M^{A} \rightarrow 0 . \Lambda \in M_{A}$ is projective if $(\bullet)^{A}$ is exact. $\Lambda$ is finitely presented as a right $A$-module if $\operatorname{Ker} \mathcal{X}$ is a finitely generated right ideal of $A$. We now give some particular examples of ring morphisms with a grouplike functional. More examples can be found in [6].

EXAMPLE 3.1. Let $H$ be a Hopf algebra over a commutative ring $k$, and $\Lambda$ a left $H$ module algebra. The smash product $A=\Lambda \# H$ is equal to $\Lambda \otimes H$ as a $k$-module, with multiplication defined by

$$
(\lambda \# h)(\mu \# k)=\lambda\left(h_{(1)} \cdot \mu\right) \# h_{(2)} k .
$$


Let $\eta_{H}$ and $\varepsilon_{H}$ be the unit and counit of $H$. Then $i=I_{\Lambda} \otimes \eta_{H}: \Lambda \rightarrow \Lambda \# H$ is a ring morphism, and $\chi=I_{\Lambda} \otimes \varepsilon_{H}: \Lambda \# H \rightarrow \Lambda$ is a left grouplike functional. It is easy to compute that $B=\Lambda^{A}=\Lambda^{H}$ is the subring of invariants of $\Lambda$. Thus we find necessary and sufficient conditions for projectivity and flatness of a module over the subring of invariants, and we recover the results obtained by the second author in [10].

In the particular situation where $H=k G$ is a group ring and $\Lambda$ is a $G$-module algebra, $A$ is a skew group ring, and we recover [8, Theorems 3 and 5], in which necessary and sufficient conditions for projectivity and flatness of $\Lambda$ as a $\Lambda^{G}$-module are given.

This example can be extended to factorization structures; we refer to [6] for details.

EXAMPLE 3.2. Let $j: S \rightarrow \Lambda$ be a morphism of algebras over a commutative ring $k$. Then $i: \Lambda \rightarrow A=S^{\text {op }} \otimes \Lambda, i(\lambda)=1_{S} \otimes \lambda$ is a ring morphism, and $\chi: A \rightarrow \Lambda, \chi(s \otimes \lambda)=j(s) \lambda$ is a right grouplike functional. We easily compute that

$$
B=\Lambda^{A}=\{b \in \Lambda \mid b j(s)=j(s) b, \forall s \in S\}=\Lambda^{S}
$$

is the centralizer of $S$ in $\Lambda$. Thus we obtain necessary and sufficient conditions for projectivity and flatness over the centralizer.

EXAMPLE 3.3. Let $\Lambda$ be a ring and $\left(\mathscr{C}, \Delta_{\mathscr{G}}, \varepsilon_{\mathscr{G}}\right)$ a $\Lambda$-coring (this is a coalgebra in the monoidal category ${ }_{\Lambda} M_{\Lambda}$ ). Corings were introduced by Sweedler [14]. The language of corings allows us to generalize, unify, and formulate more elegantly many results about generalized Hopf modules. This is due to the fact that Hopf modules (and their generalizations) are in fact comodules over a coring. This observation can be found implicitly in the proof of [7, Theorem 2.11]. It is tributed to Takeuchi in a Mathematical Review (MR 2000c 16047) written by Masuoka, but the referee informs us that it was already known by Sweedler. Only recently the importance of the coring viewpoint became clear. Brzeziński's [3] is the first of a series of papers on this subject; we refer to [5] for more references and the actual state of the art.

$x \in \mathscr{C}$ is called grouplike if $\Delta_{\mathscr{C}}(x)=x \otimes_{\Lambda} x$ and $\varepsilon_{\mathscr{C}}(x)=1_{\Lambda}$ (see [4]). The left dual of $\mathscr{C}$ is then a ring: $A={ }_{\Lambda} \operatorname{Hom}(\mathscr{b}, \Lambda)$ has an associative multiplication

$$
(f \# g)(c)=g\left(c_{(1)} f\left(c_{(2)}\right)\right) .
$$

The unit is $\varepsilon_{\mathscr{c}}$. We have a ring morphism

$$
i: \Lambda \longrightarrow A, \quad i(\lambda)(c)=\varepsilon_{\mathscr{C}}(c) \lambda,
$$

and a right grouplike functional

$$
\chi: A \longrightarrow \Lambda, \quad \chi(f)=f(x)
$$

We easily compute that

$$
B=\Lambda^{A}=\{b \in \Lambda \mid b f(x)=f(x b), \forall f \in A\},
$$

and we find necessary and sufficient conditions for projectivity and flatness of a right $B$-module. 
ACKNOWLEDGMENT. This research is supported by the Project G.0278.01 "Construction and applications of noncommutative geometry: from algebra to physics" from FWO Vlaanderen.

\section{REFERENCES}

[1] T. Albu and C. Năstăsescu, Relative Finiteness in Module Theory, Monographs and Textbooks in Pure and Applied Mathematics, vol. 84, Marcel Dekker, New York, 1984.

[2] H. Bass, Algebraic K-Theory, W. A. Benjamin, New York, 1968.

[3] T. Brzeziński, The structure of corings: induction functors, Maschke-type theorem, and Frobenius and Galois-type properties, Algebr. Represent. Theory 5 (2002), no. 4, 389410.

[4] _ The structure of corings with a grouplike element, Noncommutative Geometry and Quantum Groups, Banach Center Publ., vol. 61, Polish Academy of Sciences, Warsaw, 2003, pp. 21-35.

[5] T. Brzezinski and R. Wisbauer, Corings and Comodules, London Mathematical Society Lecture Note Series, vol. 309, Cambridge University Press, Cambridge, 2003.

[6] S. Caenepeel, J. Vercruysse, and S. Wang, Morita theory for corings and cleft entwining structures, J. Algebra 276 (2004), 210-235.

[7] Y. Doi and M. Takeuchi, Hopf-Galois extensions of algebras, the Miyashita-Ulbrich action, and Azumaya algebras, J. Algebra 121 (1989), no. 2, 488-516.

[8] J. J. García and A. Del Río, On flatness and projectivity of a ring as a module over a fixed subring, Math. Scand. 76 (1995), no. 2, 179-193.

[9] V. E. Govorov, On flat modules, Sibirsk. Mat. Ž. 6 (1965), 300-304.

[10] T. Guédénon, Projectivity and flatness of a module over the subring of invariants, Comm. Algebra 29 (2001), no. 10, 4357-4376.

[11] S. Jøndrup, When is the ring a projective module over the fixed point ring?, Comm. Algebra 16 (1988), no. 10, 1971-1992.

[12] D. Lazard, Sur les modules plats, C. R. Acad. Sci. Paris 258 (1964), 6313-6316 (French).

[13] M. Sato, Fuller's theorem on equivalences, J. Algebra 52 (1978), no. 1, 274-284.

[14] M. E. Sweedler, The predual theorem to the Jacobson-Bourbaki theorem, Trans. Amer. Math. Soc. 213 (1975), 391-406.

S. Caenepeel: Faculty of Applied Sciences, Vrije Universiteit Brussel (VUB), 1050 Brussels, Belgium

E-mail address: scaenepe@vub.ac.be

T. Guédénon: Faculty of Applied Sciences, Vrije Universiteit Brussel (VUB), 1050 Brussels, Belgium

E-mail address: tguedeno@vub.ac.be 


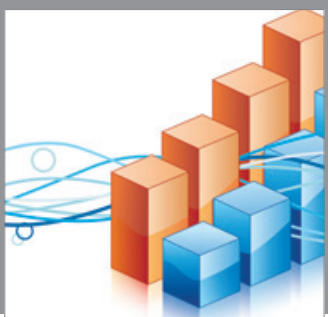

Advances in

Operations Research

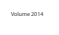

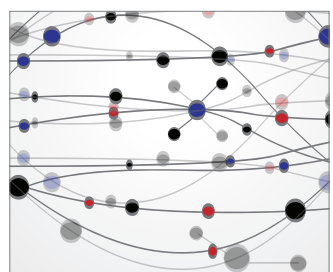

\section{The Scientific} World Journal
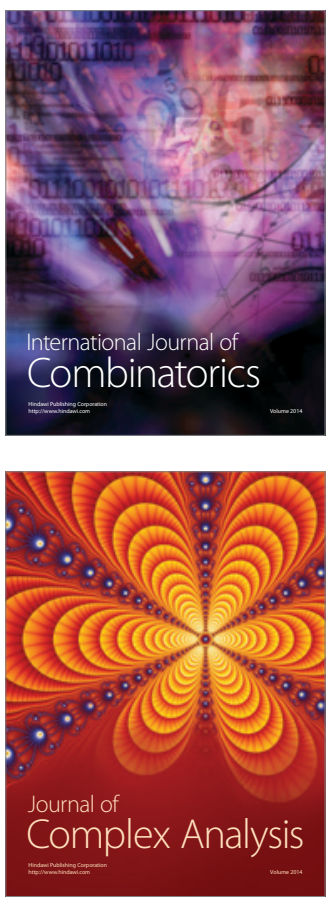

International Journal of

Mathematics and

Mathematical

Sciences
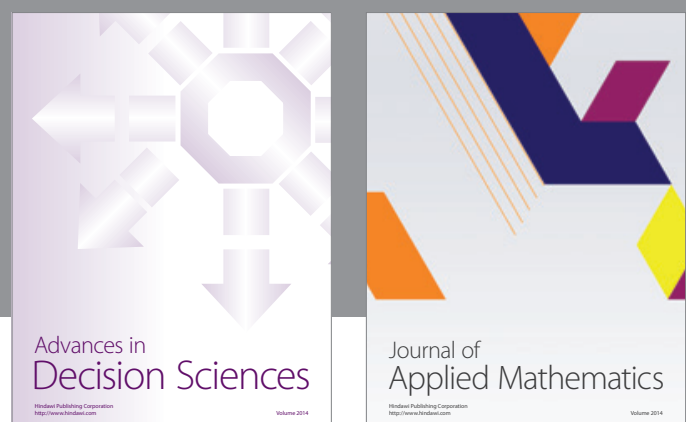

Journal of

Applied Mathematics
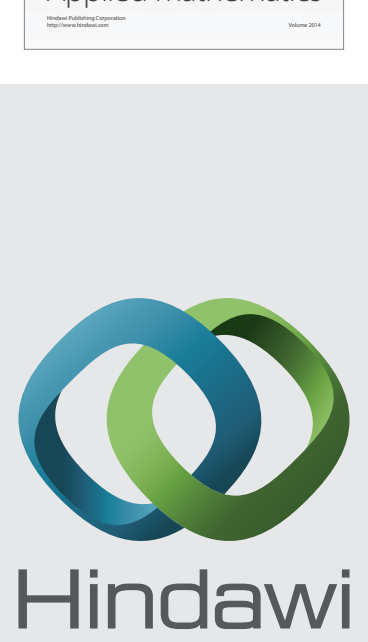

Submit your manuscripts at http://www.hindawi.com
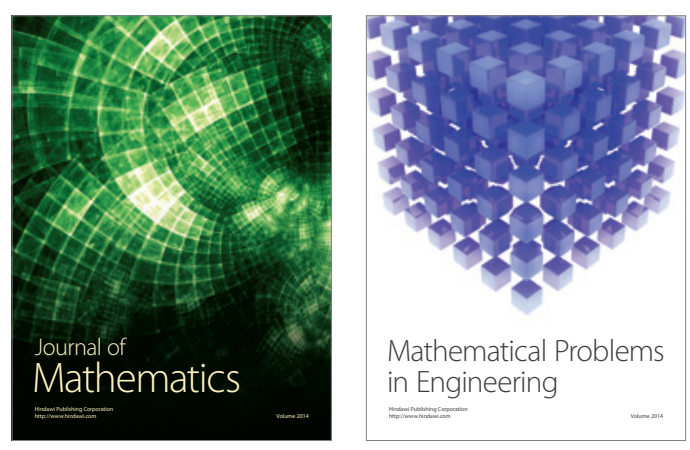

Mathematical Problems in Engineering
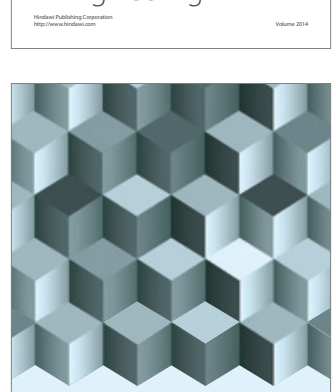

Journal of

Function Spaces
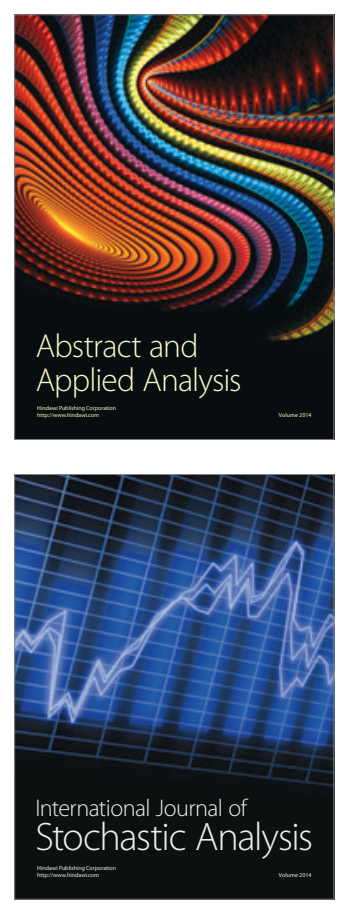

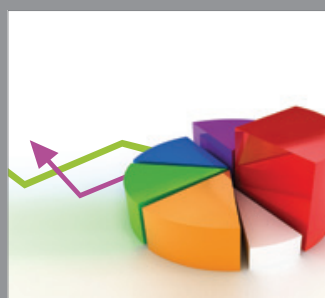

ournal of

Probability and Statistics

Promensencen
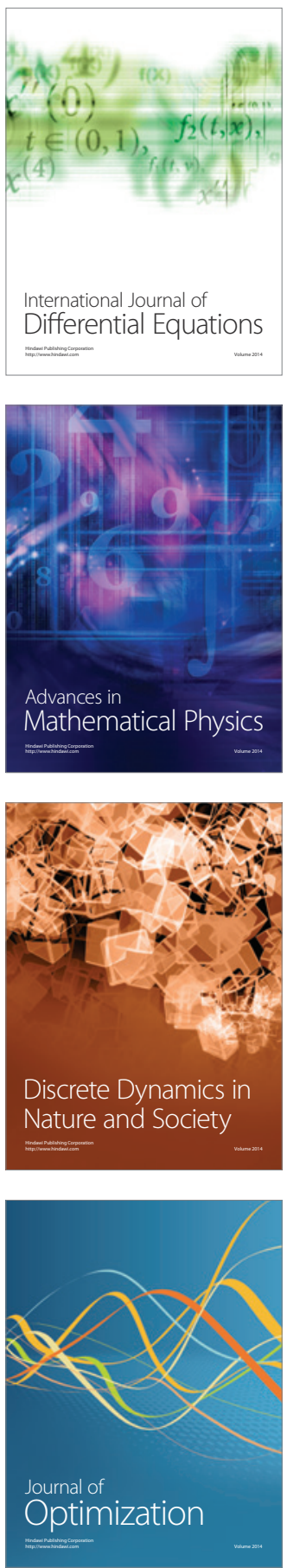